\title{
Lower bounds on waiting times for degenerate parabolic equations and systems
}

\author{
LORENZO GIACOMELLI ${ }^{\dagger}$ \\ Dipartimento Me.Mo.Mat., Università di Roma La Sapienza, \\ Via Scarpa, 16, I-00161 Roma, Italy \\ AND \\ GÜNTHER GRÜN \\ Institut für Angewandte Mathematik, Universität Bonn, \\ Beringstr. 6, D-53115 Bonn, Germany
}

[Received 1 September 2005 and in revised form 1 November 2005]

\begin{abstract}
We extend the method in [19] to obtain quantitative estimates of waiting times for free boundary problems associated with degenerate parabolic equations and systems. Our approach is multidimensional, it applies to a large class of equations, including thin-film equations, (doubly) degenerate equations of second and of higher order and also systems of semiconductor equations. For these equations, we obtain lower bounds on waiting times which we expect to be optimal in terms of scaling. This assertion is true for the porous-medium equation which seems to be the only PDE for which two-sided quantitative estimates of the waiting time have been established so far.
\end{abstract}

2000 Mathematics Subject Classification: 35B99, 35G25, 35K30, 35K50, 35K65, 35Q35, 35R35, 76D08, 76D27.

Keywords: Waiting time; nonlinear higher-order PDE; degenerate parabolic equations; degenerate parabolic systems; thin-film equations; porous-medium equations; free boundary problems; lubrication theory.

\section{Introduction}

It is one of the most intriguing features of free boundary problems associated with degenerate parabolic equations and systems that solutions may exhibit so-called "waiting time phenomena". Generally speaking, this means that for some time the solution's support locally does not expand, or shrink, or both, at a point $x_{0}$ of its boundary. There are different reasons for waiting time phenomena to occur-in most cases they come as a consequence of initial data being sufficiently flat in a neighborhood of $x_{0}$. Here we shall be concerned with a "forward" waiting time $T^{*}$ : this is the time it takes before the solution's support begins to expand over $x_{0}$ - until that time, it may either stand still or even recede from $x_{0}$. The precise notion is given by the following definition.

DEFinition 1.1 We say a function $w: \mathbb{R}^{N} \times[0, \infty)$ has positive waiting time at $x_{0} \in \partial \operatorname{supp}\left(w_{0}\right)$ if there exists $T>0$ such that $x_{0} \in \overline{\mathbb{R}^{N} \backslash \operatorname{supp}(w(\cdot, t))}$ for almost all $t \in[0, T)$. In this case, the

\footnotetext{
†-mail: giacomelli@dmmm.uniroma1.it

‡E-mail: gg@iam.uni-bonn.de
} 
waiting time $T^{*}$ is defined by

$$
T^{*}=\sup \left\{T>0: x_{0} \in \overline{\mathbb{R}^{N} \backslash \operatorname{supp}(w(\cdot, t))} \text { for a.e. } t \in[0, T)\right\} .
$$

Here, the support of a function $w(\cdot, t)$ is understood as the complement of the union of all open subsets of $\mathbb{R}^{N}$ on which $w(\cdot, t)=0$ almost everywhere with respect to the Lebesgue measure. Though in most applications $w$ is non-negative, in some of them it may change sign (cf. for instance (3.3) below), and Definition 1.1 covers these cases, too.

In recent years, much effort has been invested to formulate sufficient conditions for the occurrence of waiting time phenomena for various degenerate parabolic equations. For second order equations and systems, the techniques range from comparison principles (see Knerr [31] for the porous-medium equation) to Harnack-type inequalities (see the optimal result of Alikakos [1] on the porous-medium equation, which in turn relies on [7] and [10]) and to various energy methods (see Antontsev [3], Antontsev and Díaz [4], and Antontsev, Díaz, and Shmarev [6] for second order operators, and Díaz, Galiano, and Jüngel [23] for a degenerate system of semiconductor equations). To analyze also higher order equations, energy methods based on the analysis of functional inequalities and iterative procedures have been introduced by Shishkov [35] and applied also to waiting time phenomena by Shishkov and Shchelkov [36] for a class of quasilinear degenerate equations not including thin-film-type equations (see also the references in [25]). Recently, in studying waiting time phenomena for thin-film equations, Dal Passo and the authors of this paper [19] have proposed the use of cone-shaped test functions and a simplification of these methods through an extension of the classical Stampacchia lemma [37], which has been shown in [20] to be applicable to the porous-medium equation, higher order doubly degenerate equations of the form (3.3) below, and other thin-film-type equations. Cone-supported test functions were also used in [34], independently of [19], to study the finite speed of propagation property for higher order operators with convection.

Not as much has been achieved regarding estimates on the size of waiting times. With the exception of [36], neither the aforementioned papers nor the recent monograph by Antontsev, Díaz, and Shmarev [5] provide any result on that problem. The only two-sided estimates established so far pertain to the porous-medium equation

$$
u_{t}=\Delta g(u), \quad g(u) \geqslant C u^{m}, \quad m>1 .
$$

In one space dimension, and for $g(u)=u^{m}$, Aronson, Caffarelli, and Kamin [8] have shown that the waiting time $T^{*}$ is estimated by

$$
K B^{1-m} \leqslant T^{*} \leqslant K A^{1-m}, \quad K=\frac{m^{2}-1}{2 m} \quad \text { for } N=1,
$$

provided the initial data satisfy

$$
\lim _{x \downarrow x_{0}} \frac{u_{0}(x)}{\left|x-x_{0}\right|^{2 / m-1}}=A \quad \text { and } \quad u_{0}(x) \leqslant B\left(x-x_{0}\right)_{+}^{2 /(m-1)} .
$$

In [17], Chipot and Sideris have extended the upper bound part of (1.1) to the higher dimensional case: in particular, their result implies that if the initial support has an "interior cone property" at $x_{0} \in \partial \operatorname{supp}\left(u_{0}\right)$, then

$$
u_{0}(x) \geqslant A\left|x-x_{0}\right|^{2 /(m-1)} \Rightarrow T^{*} \leqslant C A^{1-m} \quad \text { for } N \geqslant 1 .
$$

Looking carefully at the proof of Proposition 4.2 in Alikakos' paper [1], it becomes evident that 
Harnack-type inequalities permit deducing a lower bound on the waiting time for the porousmedium equation also in the multi-dimensional setting; in particular,

$$
u_{0}(x) \leqslant B\left|x-x_{0}\right|^{2 /(m-1)} \Rightarrow T^{*} \geqslant C B^{1-m} .
$$

It seems, however, that this observation has never been written down. Comparing (1.3) with (1.2), we see that the scaling with respect to the constant $B$ is optimal. In [36], Shishkov and Shchelkov have obtained estimates on the size of the waiting time for a class of quasilinear degenerate equations of arbitrary order not including thin-film-type equations. For the porous-medium equation in one space dimension, they recover the optimal lower bound in the sense of (1.3). Due to their choice of test function, however, in the multi-dimensional case their result only applies to convexity points of $\partial \operatorname{supp}\left(u_{0}\right)$, and seems not to imply 1.3 at points where this boundary is smooth ${ }^{1}$

In the present paper, we prove new lower bounds on the size of waiting times. The strategy extends the one in [19] and is based on energy/entropy estimates instead of Harnack-type inequalities. It not only permits one to recover the optimal result given by (1.3) for solutions of the porous-medium equation, but has in addition the advantage of being applicable to a wide class of degenerate evolution operators, including the porous-medium equation, higher order doubly degenerate equations of the form (3.3) below, other thin-film-type equations and also systems, and to include non-convexity points of the initial support.

Let us describe the outline of the paper. Our starting point is the following, rather general class of integral estimates ${ }^{2}$

$$
\int_{\mathbb{R}^{N}} \zeta^{l p}|w(\cdot, T)|^{q}+C_{0}^{-1} \int_{0}^{T} \int_{\mathbb{R}^{N}} \zeta^{k p}\left|D^{k} w\right|^{p} \leqslant \int_{\mathbb{R}^{N}} \zeta^{k p} F\left(w_{0}\right)+C_{0} \int_{0}^{T} \int_{[\zeta>0]}|w|^{s} .
$$

Estimate (1.4) applies in particular to solutions of the Cauchy problem for all the aforementioned applications. In Section 2, we will prove an abstract result on lower bounds for waiting times, Theorem 2.1. In contrast to the occurrence of the waiting time phenomenon, which purely depends on the flatness of initial data near the free boundary point under consideration, its duration is estimated by a global quantity, which depends on the overall shape of the initial datum (in fact, it may also be influenced by boundary conditions, a topic that we shall not consider here).

The proof of Theorem 2.1 can be sketched as follows. Using the ingredients of [19], i.e. localization functions supported on cones, interpolation arguments and a new version of Stampacchia's iteration lemma, we identify the boundedness of

$$
S(R)=\sup _{r \in(0, R)} r^{-\gamma-N} \int_{\mathcal{C}\left[x_{0}+r a, \theta, a\right]} F\left(w_{0}\right)
$$

as $R \downarrow 0$ to be a sufficient criterion for the occurrence of a waiting time phenomenon in the sense of Definition 1.1. Here, $\mathcal{C}\left[x_{0}+r a, \theta, a\right]$ is a half-cone with vertex at $x=x_{0}+r a$, opening angle $\theta$ and symmetry axis parallel to $a$ (see Figure 11. Taking for $\gamma$ the optimal coefficient for the occurrence of a waiting time phenomenon, a closer look at the modified Stampacchia lemma reveals the waiting time $T^{*}$ to depend on the parameter $R>0$. Optimizing with respect to $R$ gives the desired lower bound.

1 Note that [36] also deals with absorption terms, and that the paper of A. Shishkov in Differential Equations 29 (1993), $460-469$, introduces some of the tools used there.

${ }^{2}$ Here $k, l, p, q, s$ are appropriate real numbers, $\zeta$ is a weight function supported on a cone, and $F: W^{k}\left(\mathbb{R}^{N}\right) \rightarrow$ $L_{\text {loc }}^{1}\left(\mathbb{R}^{N} ; \mathbb{R}_{0}^{+}\right)$is a non-negative differential operator; see Theorem 2.1 for the precise formulation of the assumptions. 

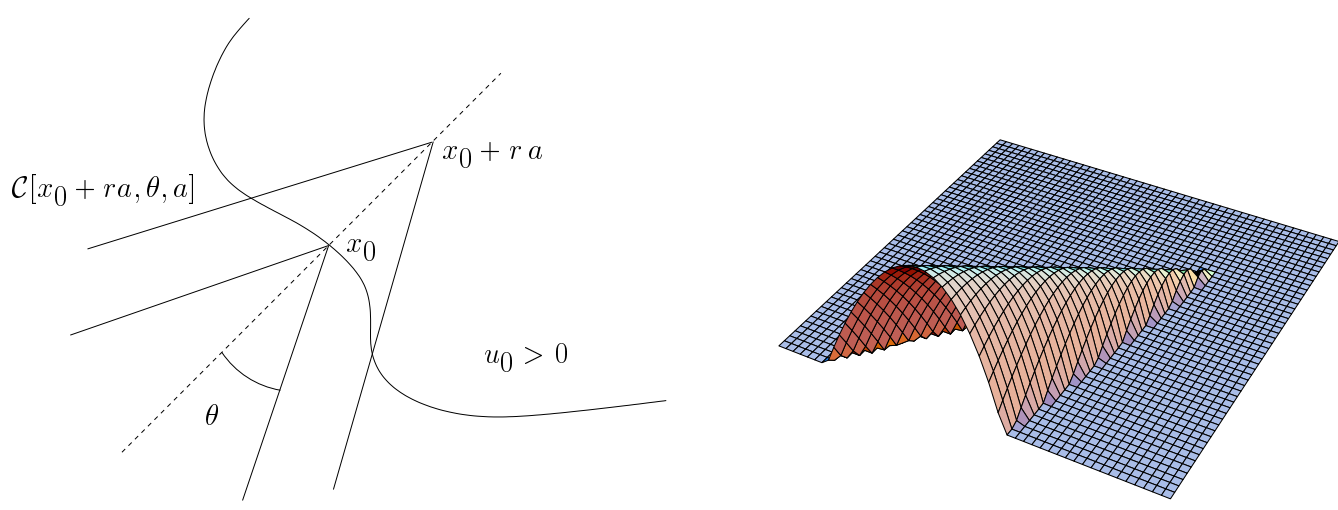

FIG. 1. The cones $\mathcal{C}\left[x_{0}+r a, \theta, a\right]$ intruding the initial support at $x_{0}$, and the cone-shaped test function supported there.

Section 3 is devoted to the application of Theorem 2.1 to specific examples. For the porousmedium equation, we recover the scaling result of (1.3) in the multi-dimensional case. Similarly, we obtain a lower bound of the waiting time for the thin-film equation

$$
u_{t}+\operatorname{div}\left(u^{n} \nabla \Delta u\right)=0
$$

in space dimensions $N \in\{1,2,3\}$ and for $n \in(0,3)$. Note that for $n \geqslant 3$ solutions are expected to have support constant in time. In one spatial dimension, a similar result is presented for doubly degenerate thin-film equations as they are used to model wetting of surfaces by shear-thinning power-law fluids. Subsection 3.1 terminates with an application to doubly degenerate parabolic equations of arbitrary order.

To demonstrate the applicability of Theorem 2.1 to systems of reaction-diffusion equations, in Section 4 we consider a system of degenerate semiconductor equations for which the occurrence of a waiting time phenomenon was investigated previously by Díaz, Galiano, and Jüngel [23]. Here, in addition to a quantitative lower bound, we obtain improvements of the conditions on the nonlinearity, on the flatness of initial data, and on the growth rate of the recombination term (see Section 4 for more details on comparison with [23]).

Summarizing, in this paper we present a general recipe to establish quantitative lower bounds on waiting times; we show that it applies to problems for which no such bound has been known so far, and that it gives the optimal bound in terms of scaling - at least in those cases for which the upper bound is already established.

Let us conclude by pointing out a couple of open questions. The first one is of course optimality of the lower bound-in fact, even a non-sharp upper bound would be of interest. The second one is of a more technical nature: As we shall see in Sections 3 and 4 , the application of Theorem 2.1 to most of the aforementioned examples yields a lower bound on $T^{*}$ which depends on an $L^{q}$-norm of the initial datum, with $q$ greater than one (though sometimes arbitrarily close to one); it is reasonable to expect, and worth seeking for, analogous results in terms of the $L^{1}$-norm. For the porous-medium equations, both questions have been solved by duality arguments, whose application may turn out to be fairly difficult in more general cases due to the lack of comparison arguments. Finally, it would be interesting to investigate the quantitative effect of boundary conditions on the size of the waiting time. 


\section{The abstract result}

The abstract result requires some preliminary remarks on notation. For $x_{0} \in \mathbb{R}^{N}, a \in S^{N-1}$ and $\theta \in(0, \pi / 2)$, consider the functions

$$
\zeta\left[x_{0}, \theta, a\right](x):=\left\langle x_{0}-x, a\right\rangle_{+}\left(1-\frac{\left|x_{0}-x-\left\langle x_{0}-x, a\right\rangle a\right|^{2}}{\left\langle x_{0}-x, a\right\rangle^{2} \tan ^{2} \theta}\right)_{+} .
$$

Here $(x)_{+}$denotes $\max \{x, 0\}$. These functions are supported on half-cones

$$
\mathcal{C}\left[x_{0}, \theta, a\right]=\operatorname{supp}\left(\zeta\left[x_{0}, \theta, a\right]\right) \subset \mathbb{R}^{N}
$$

with vertex $x_{0} \in \mathbb{R}^{N}$, opening angle $\theta$, and axis parallel to $a$ in such a way that $x_{0}+a \notin \mathcal{C}\left[x_{0}, \theta, a\right]$. Similarly, we introduce the following one-parameter family of admissible test functions:

$$
\mathcal{F}\left[x_{0}, \theta, a\right]=\left\{\zeta\left[x_{0}+r a, \theta, a\right](\cdot): r \geqslant 0\right\} .
$$

Let us introduce furthermore for $k \in \mathbb{N}$ continuous functions

$$
H: \mathbb{R} \times \mathbb{R}^{N} \times \mathbb{R}^{N^{2}} \times \cdots \times \mathbb{R}^{N^{k}} \rightarrow \mathbb{R}_{0}^{+}, \quad H(0)=0 .
$$

Associated with $H$, we consider the operator

$$
F: W_{\text {loc }}^{k, 1}\left(\mathbb{R}^{N}\right) \rightarrow L_{\text {loc }}^{1}\left(\mathbb{R}^{N} ; \mathbb{R}_{0}^{+}\right)
$$

defined by

$$
F(w)(x):=H\left(w(x), D w(x), \ldots, D^{k} w(x)\right) .
$$

The abstract result reads as follows.

THEOREM 2.1 (Lower bound on waiting time) Let $l, p, q, s \in \mathbb{R}$ and $k \in \mathbb{N}$ satisfy

$$
0<l \leqslant k, \quad 0<q<s, \quad p \geqslant \max \left\{\frac{N s}{N+k q}, 1\right\},
$$

and let $F$ be a given non-negative differential operator as in 2.3. Assume that a function $w$ satisfies the following:

- for some $T_{0}<\infty$,

$$
\int_{\mathbb{R}^{N}} F\left(w_{0}\right)<\infty, \quad \int_{0}^{T_{0}} \int_{\mathbb{R}^{N}}|w|^{s}<\infty ;
$$

- $\operatorname{supp}(w(\cdot, 0))=\operatorname{supp}\left(w_{0}\right)$ has the "exterior cone property" at $x_{0} \in \partial \operatorname{supp}\left(w_{0}\right)$, i.e. $\operatorname{supp}\left(w_{0}\right) \cap$ $\operatorname{supp}\left(\zeta\left[x_{0}, \theta, a\right]\right)=\emptyset$ for some $\theta \in(0, \pi / 2)$ and $a \in S^{N-1}$;

- there exists a positive constant $C_{0}$ such that

$$
\int_{\mathbb{R}^{N}} \zeta^{l p}|w(\cdot, T)|^{q}+C_{0}^{-1} \int_{0}^{T} \int_{\mathbb{R}^{N}} \zeta^{k p}\left|D^{k} w\right|^{p} \leqslant \int_{\mathbb{R}^{N}} \zeta^{k p} F\left(w_{0}\right)+C_{0} \int_{0}^{T} \int_{[\zeta>0]}|w|^{s}
$$

for all $T \in\left(0, T_{0}\right)$ and all $\zeta \in \mathcal{F}\left[x_{0}, \theta, a\right]$. 
Let

$$
S(R)=\sup _{r \in(0, R)} r^{-\gamma-N} \int_{\mathcal{C}\left(x_{0}+r a, \theta, a\right)} F\left(w_{0}\right), \quad \gamma=-k p+\frac{l[N(p-s)+k p s]}{k(s-q)} .
$$

Then the following holds true.

(1) If

$$
\lim _{R \downarrow 0} S(R)<\infty,
$$

then $w$ has positive waiting time at $x_{0}$ in the sense of Definition 1.1

(2) if in addition

$$
S=\sup _{R>0} S(R)<\infty,
$$

then the waiting time $T^{*}$ at $x_{0}$ is estimated from below by

$$
T^{*} \geqslant C S^{-\frac{k p(s-q)}{k p q+N(p-s)}},
$$

where $C$ is a positive constant which only depends on $C_{0}, \theta, N, k, l, p, q$, and $s$.

Part (1) recovers an earlier result of Dal Passo and the authors [19, 20]. It is a qualitative result: The occurrence of a waiting time phenomenon only depends on the local behavior of the initial datum at the point of interest. On the other hand, part (2) extends [20] and is a quantitative result: The duration of the waiting time phenomenon is estimated by a global quantity, determined by the overall shape of the initial datum.

REMARK 2.2 If the opening angle $\theta$ is chosen so that $\operatorname{supp}\left(w_{0}\right) \cap \operatorname{supp}\left(\zeta\left[x_{0}, 2 \theta, a\right]\right)=\emptyset$, then Theorem 2.1 holds true with $S(R)$ in 2.8 and (2.9) replaced by

$$
\bar{S}(R)=\sup _{r \in(0, R)} r^{-\gamma-N} \int_{B\left(x_{0}, r\right)} F\left(w_{0}\right) .
$$

Indeed, in this case an elementary geometric argument (see [19, Fig. 1]) implies that

$$
\operatorname{supp}\left(w_{0}\right) \cap \mathcal{C}\left[x_{0}+r a, \theta, a\right] \subset B\left(x_{0}, r\right),
$$

so that $S(R) \leqslant \bar{S}(R)$.

Remark 2.2 allows one to infer explicit versions of Theorem 2.1 in the case of power-type behavior of the initial datum in a straightforward way. The following prototypical case occurs in many applications.

COROllary 2.3 Let $\mu>0$. Assume that $w=|u|^{\mu-1} u$ satisfies the assumptions of Theorem 2.1 with $(l, s)=(k, p)$ and $F\left(w_{0}\right)=\left|w_{0}\right|^{q}$. Then:

(1) A critical growth exponent $\gamma^{*}=k p / \mu(p-q)$ is identified for the function $u$, such that if

$$
\lim _{x \rightarrow x_{0}} \frac{\left|u_{0}(x)\right|}{\left|x-x_{0}\right|^{\gamma^{*}}}<\infty
$$

then $T^{*}>0$; 
(2) a positive constant $C=C\left(C_{0}, \theta, N, \mu, k, p, q, s\right)$ exists such that if in addition

$$
\left|u_{0}(x)\right| \leqslant B\left|x-x_{0}\right|^{\gamma^{*}},
$$

then

$$
T^{*} \geqslant C B^{-\mu(p-q)} \text {. }
$$

In the applications to be presented in Sections 3 and 4 , the constant $C_{0}$ does not depend on initial data, but does depend on $\theta$. The opening angle $\theta$ plays indeed a crucial role, since both parts (1) and (2) are sensitive to the shape of the support. For instance, the critical growth exponent $\gamma^{*}$ improves if the initial support has an outward-pointing cusp at $x_{0}$.

Our strategy is based on three main ingredients. The first ingredient is a calculus lemma which extends the classical Stampacchia lemma and gives sufficient conditions for a non-negative, nonincreasing function to become zero at a given point. Its proof is a straightforward modification of one presented in [19]. The lemma reads as follows.

LEMmA 2.4 Assume that a non-negative, non-increasing function $G:[0, R] \rightarrow[0, \infty)$ is such that

$$
G(\xi) \leqslant \frac{K}{(\xi-\eta)^{\alpha}}\left\{G(\eta)+\tilde{S} \cdot(R-\eta)^{\alpha /(\beta-1)}\right\}^{\beta}
$$

for all $0 \leqslant \eta<\xi \leqslant R$, where $K>0, \tilde{S} \geqslant 0, \alpha>0$ and $\beta>1$ are real constants. If in addition

$$
R^{\alpha /(\beta-1)} \geqslant\left(2^{\beta(\alpha+\beta-1) /(\beta-1)} K\right)^{1 /(\beta-1)}\left\{G(0)+\tilde{S} \cdot R^{\alpha /(\beta-1)}\right\},
$$

then $G(R)=0$.

The second ingredient is the particular choice of test functions already introduced in 2.2. Their cone-shaped positivity set allows one to handle non-convex initial supports and is such that homogeneous interpolations hold true; furthermore, they are well-behaved with respect to nesting:

LEMma 2.5 The functions $\zeta\left[x_{0}+r a, \theta, a\right](x)$ given by $[2.1)$ are such that for all $r>0$ :

$$
\begin{array}{ll}
\zeta\left[x_{0}+r a, \theta, a\right](x) \geqslant r & \text { for all } x \in \mathcal{C}\left[x_{0}, \theta, a\right], \\
\zeta\left[x_{0}+r a, \theta, a\right](x) \leqslant 2 r & \text { for all } x \in \mathcal{C}\left[x_{0}+r a, \theta, a\right] \backslash \mathcal{C}\left[x_{0}, \theta, a\right] .
\end{array}
$$

The last ingredient is an interpolation argument, which-thanks to the aforementioned properties of the test functions - transforms the integral inequality 2.6 into a pointwise inequality of the form 2.11, for the integral function

$$
G_{T, R}(r)=\int_{0}^{T} \int_{\mathcal{C}\left[x_{0}+(R-r) a, \theta, a\right]}|w|^{s},
$$

to which Lemma 2.4 can then be applied. It leads to the following result.

LEMma 2.6 Under the assumptions of Theorem 2.1. there exists a constant $C_{1}=$ $C_{1}\left(C_{0}, \theta, N, p, q, s, k, l\right)$ such that

$$
G_{T, R}(\xi) \leqslant \frac{\left(C_{1} T\right)^{1-\vartheta}}{(\xi-\eta)^{\alpha}}\left\{G_{T, R}(\eta)+S(R)(R-\eta)^{\alpha /(\beta-1)}\right\}^{\beta}
$$


for all $T \in\left(0, T_{0}\right)$ and all $0 \leqslant \eta<\xi \leqslant R$, with $S(R)$ given by 2.7) and with parameters

$$
\vartheta=\frac{N(s-q)}{k p q+N(p-q)}, \quad \beta=\vartheta+(s-\vartheta p) \frac{1}{q}, \quad \alpha=l p \beta+(k-l) p \vartheta .
$$

The combination of Lemma 2.4 and Lemma 2.6 yields Theorem 2.1. as we now show.

Proof of Theorem 2.1 In order to apply Lemma 2.4 with $G=G_{T, R}$ and $\tilde{S}=S(R)$, we only need to ensure that

$$
R^{\alpha /(\beta-1)} \geqslant\left(C_{2} T\right)^{(1-\vartheta) /(\beta-1)}\left\{G_{T, R}(0)+S(R) R^{\alpha /(\beta-1)}\right\}
$$

for some $T \in\left(0, T_{0}\right)$ and $R>0$ (here $\left.C_{2}=2^{\beta(\alpha+\beta-1) /(1-\vartheta)(\beta-1)} C_{1}\right)$. Then it follows from Lemmas 2.4 and 2.6 that $G_{T, R}(R)=0$, which means that we have a waiting time phenomenon of duration $T$ at $x_{0}$. To establish part (1), we just observe that 2.16 is satisfied by all $R$ and $T$ sufficiently small provided 2.8 holds. To establish part (2), we proceed as follows. Writing 2.16 equivalently as

$$
T \leqslant C_{2}^{-1}\left(R^{-\alpha /(\beta-1)} G_{T, R}(0)+S(R)\right)^{-(\beta-1) /(1-\vartheta)} .
$$

we see that we have a waiting time phenomenon of duration $T$ at $x_{0}$ in particular if

$$
T \leqslant C_{2}^{-1}\left(R^{-\alpha /(\beta-1)} G_{T, R}(0)+\sup _{\rho>0} S(\rho)\right)^{-(\beta-1) /(1-\vartheta)}
$$

for some $R>0$. Taking the supremum on the right-hand side with respect to $R$ and using 2.5 yields

$$
T^{*} \geqslant C_{2}^{-1} S^{-(\beta-1) /(1-\vartheta)}
$$

as the desired lower bound for waiting time. Finally, we notice that

$$
\frac{\beta-1}{1-\vartheta}=\frac{k p(s-q)}{k p q+N(p-s)},
$$

which entails 2.10 and proves Theorem 2.1

The rest of the section is concerned with the proofs of Lemmas 2.5 and 2.6

Proof of Lemma 2.5. Without loss of generality we may let $x_{0}=0$ and $a=e_{N}$. We introduce for notational convenience the projection $\bar{x}_{N}=x-\left\langle x, e_{N}\right\rangle e_{N}$, the sets

$$
\mathcal{C}(r)=\mathcal{C}\left[r e_{N}, \theta, e_{N}\right]
$$

and the functions

$$
\zeta_{r}=\zeta\left[r e_{N}, \theta, e_{N}\right]
$$

so that

$$
\zeta_{r}(x)=\left(r-x_{N}\right)_{+}\left(1-\frac{\left|\bar{x}_{N}\right|^{2}}{\left(r-x_{N}\right)^{2} \tan ^{2} \theta}\right)_{+} .
$$

Since $\zeta_{r}$ is decreasing with respect to $\left|\bar{x}_{N}\right|$, in order to estimate it from below on $\mathcal{C}(0)$, respectively from above in $\mathcal{C}(r) \backslash \mathcal{C}(0)$, we only need to evaluate it at $\partial \mathcal{C}(0)=\left\{-\bar{x}_{N}=\left|x_{N}\right| \tan \theta\right\}$. There, we have

$$
\left.\zeta_{r}\right|_{\partial \mathcal{C}(0)}=r\left(\frac{r-2 x_{N}}{r-x_{N}}\right),
$$

which is decreasing for $x_{N} \in(-\infty, 0)$. Hence the bounds. 
Before giving the proof of Lemma 2.6 let us state a version of Gagliardo-Nirenberg's inequality [24, 32, 33] which will be needed in the sequel.

THEOREM 2.7 (Gagliardo-Nirenberg) Let $0<q<p, 1 \leqslant r \leqslant \infty, m \in \mathbb{N}, m>0$. Let $\mathcal{C} \subset \mathbb{R}^{N}$ be a half-cone with opening angle $\theta \in(0, \pi / 2)$. Then there exists a positive constant $C_{1}$ (depending on $N, \theta, m, p, q, r)$ such that

$$
\left(\int_{\mathcal{C}}|u|^{p}\right)^{1 / p} \leqslant C_{1}\left(\int_{\mathcal{C}}\left|D^{m} u\right|^{r}\right)^{\vartheta / r}\left(\int_{\mathcal{C}}|u|^{q}\right)^{(1-\vartheta) / q}
$$

where

$$
\frac{1}{p}=\vartheta\left(\frac{1}{r}-\frac{m}{N}\right)+(1-\vartheta) \frac{1}{q}
$$

with $\vartheta$ in the interval $[0,1)$.

Note that the summability exponents $p, q$ are allowed to be less than one; for a proof of the corresponding inhomogeneous version valid on cubes (and via linear transformation also on parallelepipeds), see [21]. From the inhomogeneous inequality, the homogeneous one follows as usual by a simple scaling argument.

Proof of Lemma 2.6 As in the proof of Lemma 2.5, we let without loss of generality $x_{0}=0$ and $a=e_{N}$. We use the abbreviations (2.17)-(2.18) and denote by $C$ a generic constant which depends on $C_{0}, \theta$, and on the parameters $N, p, q, s, k, l$. The starting point is estimate 2.6), written for $0 \leqslant \eta<\xi \leqslant R$ and $\zeta=\zeta_{R-\eta}$ in the form

$$
\begin{aligned}
\sup _{t \in(0, T)} \int_{\mathcal{C}(R-\eta)} \zeta_{R-\eta}^{l p}|w(\cdot, t)|^{q}+\int_{0}^{T} & \int_{\mathcal{C}(R-\eta)} \zeta_{R-\eta}^{k p}\left|D^{k} w\right|^{p} \\
& \leqslant C\left\{\int_{\mathcal{C}(R-\eta)} \zeta_{R-\eta}^{k p} F\left(w_{0}\right)+\int_{0}^{T} \int_{\mathcal{C}(R-\eta)}|w|^{s}\right\} .
\end{aligned}
$$

If we observe that, by 2.12 ,

$$
\left.\zeta_{R-\eta}\right|_{\mathcal{C}(R-\xi)} \geqslant(\xi-\eta) \quad \text { for } 0 \leqslant \eta<\xi \leqslant R,
$$

(2.20) simplifies to

$$
\begin{aligned}
\sup _{t \in(0, T)} \int_{\mathcal{C}(R-\xi)}|w(\cdot, t)|^{q}+ & (\xi-\eta)^{(k-l) p} \int_{0}^{T} \int_{\mathcal{C}(R-\xi)}\left|D^{k} w\right|^{p} \\
& \leqslant \frac{C}{(\xi-\eta)^{l p}}\left\{\int_{\mathcal{C}(R-\eta)} \zeta_{R-\eta}^{k p} F\left(w_{0}\right)+\int_{0}^{T} \int_{\mathcal{C}(R-\eta)}|w|^{s}\right\} .
\end{aligned}
$$

Using the homogeneous Gagliardo-Nirenberg inequality (2.19) together with the lower bound on $p$ expressed in 2.4), we may estimate

$$
\int_{0}^{T} \int_{\mathcal{C}(R-\xi)}|w|^{s} \leqslant C T^{1-\vartheta}\left(\sup _{t \in(0, T)} \int_{\mathcal{C}(R-\xi)}|w(\cdot, t)|^{q}\right)^{(s-\vartheta p) / q}\left(\int_{0}^{T} \int_{\mathcal{C}(R-\xi)}\left|D^{k} w\right|^{p}\right)^{\vartheta}
$$


with $\vartheta=\frac{N(s-q)}{k p q+N(p-q)}$. Observe that 2.21 implies the estimate

$$
\int_{0}^{T} \int_{\mathcal{C}(R-\xi)}\left|D^{k} w\right|^{p} \leqslant \frac{C}{(\xi-\eta)^{k p}}\left\{\int_{\mathcal{C}(R-\eta)} \zeta_{R-\eta}^{k p} F\left(w_{0}\right)+\int_{0}^{T} \int_{\mathcal{C}(R-\eta)}|w|^{s}\right\} .
$$

Together with 2.22) and 2.21], this entails

$\int_{0}^{T} \int_{\mathcal{C}(R-\xi)}|w|^{s} \leqslant C \frac{T^{1-\vartheta}}{(\xi-\eta) \frac{l p(s-\vartheta p)+k p q \vartheta}{q}}\left\{\int_{\mathcal{C}(R-\eta)} \zeta_{R-\eta}^{k p} F\left(w_{0}\right)+\int_{0}^{T} \int_{\mathcal{C}(R-\eta)}|w|^{s}\right\}^{(s-\vartheta p) / q+\vartheta}$.

Introducing

$$
\begin{aligned}
\beta & :=\frac{s-\vartheta p}{q}+\vartheta, \\
\alpha & :=\frac{l p(s-\vartheta p)+k p q \vartheta}{q}=l p \beta+(k-l) p \vartheta,
\end{aligned}
$$

and recalling the definition 2.14 of $G_{T, R}$, we find

$$
G_{T, R}(\xi) \leqslant \frac{C T^{1-\vartheta}}{(\xi-\eta)^{\alpha}}\left\{\int_{\mathcal{C}(R-\eta)} \zeta_{R-\eta}^{k p} F\left(w_{0}\right)+G_{T, R}(\eta)\right\}^{\beta} .
$$

Using the exterior cone property of $w_{0}$ and (2.13), we see that

$$
\zeta_{R-\eta} \chi \mathcal{C}(R-\eta) \cap \operatorname{supp}\left(w_{0}\right) \leqslant \zeta_{R-\eta} \chi \mathcal{C}(R-\eta) \backslash \mathcal{C}(0) \leqslant 2(R-\eta) .
$$

Therefore, inequality $(2.23)$ implies that

$$
G_{T, R}(\xi) \leqslant \frac{C T^{1-\vartheta}}{(\xi-\eta)^{\alpha}}\left\{(R-\eta)^{k p} \int_{\mathcal{C}(R-\eta)} F\left(w_{0}\right)+G_{T, R}(\eta)\right\}^{\beta} .
$$

Introducing

$$
\tilde{S}(R)=\sup _{r \in(0, R)} r^{k p-\alpha /(\beta-1)} \int_{\mathcal{C}(r)} F\left(w_{0}\right)
$$

we obtain

$$
G_{T, R}(\xi) \leqslant \frac{C T^{1-\vartheta}}{(\xi-\eta)^{\alpha}}\left\{G_{T, R}(\eta)+(R-\eta)^{\alpha /(\beta-1)} \tilde{S}(R)\right\}^{\beta}
$$

for all $T>0$ and $0 \leqslant \eta<\xi \leqslant R$. A tedious, but straightforward calculation shows that

$$
k p-\frac{\alpha}{\beta-1}=-\gamma-N,
$$

which means that $\tilde{S}(R)=S(R)$. Therefore 2.15 holds, and the proof is complete.

\section{Applications to degenerate parabolic equations}

In this section, we will establish lower bounds on waiting times for various degenerate parabolic equations, including doubly degenerate parabolic equations of second and higher order as well as thin-film equations. Without loss of generality, we shall assume throughout this section that $x_{0}=0$ is an element of $\partial \operatorname{supp}\left(w_{0}\right)$ and that $\operatorname{supp}\left(w_{0}\right)$ satisfies the "exterior cone property" at $x_{0}$ with opening angle $2 \theta$ and symmetry axis $a=e_{N}$, and let $\mathcal{C}(r)=\mathcal{C}\left[r e_{N}, \theta, e_{N}\right]$. 


\subsection{The porous-medium and related equations}

Let us first study the propagation of the free boundary for non-negative, mass-conserving solutions to the porous-medium equation

$$
u_{t}=\Delta u^{m}
$$

in the slow-diffusion regime $m>1$. Equation (3.1) is not only the simplest degenerate parabolic equation to which our result applies. To the best of our knowledge, it is also the only equation for which two-sided bounds on the waiting time could be established so far, as discussed in the introduction. In order to apply Theorem 2.1. we only have to establish an inequality in the spirit of 2.6). Using, for positive $\alpha$ and $\zeta \in \mathcal{F}\left[0, \theta, e_{N}\right]$, the function $\zeta^{2} u^{\alpha}$ as the test function in the weak formulation of (3.1), it is straightforward that

$$
\sup _{t \in(0, T)} \int_{\mathbb{R}^{N}} \zeta^{2} u^{\alpha+1}(\cdot, t)+C^{-1} \int_{0}^{T} \int_{\mathbb{R}^{N}} \zeta^{2}\left|D u^{(\alpha+m) / 2}\right| \leqslant \int_{\mathbb{R}^{N}} \zeta^{2} u_{0}^{\alpha+1}+C \int_{0}^{T} \int_{[\zeta>0]} u^{\alpha+m}
$$

for all $\alpha>0$ and all $\zeta \in \mathcal{F}\left[0, \theta, e_{N}\right]$, with a constant $C$ depending on $\alpha$ and $m$. Therefore (2.6) holds true for the choices

$$
w=u^{(\alpha+m) / 2}, \quad p=s=2, \quad q=\frac{2(\alpha+1)}{\alpha+m}, \quad k=l=1, \quad F\left(w_{0}\right)=w_{0}^{q} .
$$

For the function $S$, we obtain

$$
S(R)=\sup _{r \in(0, R)} r^{-(2(\alpha+1)) /(m-1)-N} \int_{\mathcal{C}(r)} u_{0}^{\alpha+1} .
$$

Thus, part (1) of Theorem 2.1 recovers the optimal growth exponent $\gamma^{*}=2 /(m-1)$ obtained by Alikakos [1], whereas part (2) yields the lower bound

$$
T^{*} \geqslant C\left(\sup _{R>0} S(R)\right)^{(1-m) /(\alpha+1)} .
$$

In particular, if the growth of the initial data is bounded as $u_{0}(x) \leqslant B|x|^{2 /(m-1)}$, Corollary 2.3 (with $\mu=(\alpha+m) / 2)$ translates as the lower bound

$$
T^{*} \geqslant C B^{1-m} \text {. }
$$

Note that (3.2) is independent of the spatial dimension. Moreover, the scaling in $B$ coincides with that of the upper bound obtained by Chipot and Sideris [17] (see [1.2p) and therefore it is optimal.

Similarly, Theorem 2.1 can be applied to doubly non-linear coercive parabolic equations of any order of the form

$$
\left(|u|^{q-2} u\right)_{t}+(-1)^{k} \sum_{|\lambda|=k} D^{\lambda}\left(\left|D^{k} u\right|^{p-2} D^{\lambda} u\right)=0
$$

with $k \geqslant 1$ and $1<q<p$. We refer e.g. to [12] for the existence theory, to [13, 9] for the finite speed of propagation property, and to [20,36] for results on waiting time. In particular (see [20]), it is possible to show that for $\zeta \in \mathcal{F}\left[0, e_{N}, \theta\right]$,

$$
\int_{\mathbb{R}^{N}} \zeta^{k p}|u(\cdot, t)|^{q}+C^{-1} \int_{0}^{t} \int_{\mathbb{R}^{N}} \zeta^{k p}\left|D^{k} u\right|^{p} \leqslant \int_{\mathbb{R}^{N}} \zeta^{k p}\left|u_{0}\right|^{q}+C \int_{0}^{t} \int_{[\zeta>0]}|u|^{p},
$$

which coincides with 2.6 with $w=u, s=p$ and $l=k$. Theorem 2.1 can then be applied; in particular, Corollary 2.3 holds with $\mu=1$. 


\subsection{Thin-film equations}

The thin-film equation

$$
u_{t}+\operatorname{div}\left(|u|^{n} \nabla \Delta u\right)=0
$$

with real parameters $n>0$ is probably the most prominent example of a higher order degenerate parabolic equation that implicitly defines a free boundary problem. With a grain of salt, (3.4) models the purely surface tension driven evolution of the height of a thin film of viscous liquid that spreads on a plane surface. In recent years, much effort has been invested to study its well-posedness and qualitative properties. The analysis mostly concentrated on zero-contact-angle solutions. These are non-negative, mass-conserving solutions characterized by additional regularity properties, which imply a zero slope at the free boundary $\partial \operatorname{supp}(u)$. Surprisingly, those zero-contact-angle solutions only exist for $n \in(0,3)$. For $n \geqslant 3$, the solution's support is expected to be constant in time. Another interesting and unexpected analytical feature is an $n$-dependent change in the structure of the operator which requires different methods in the parameter regimes $n \in(0,2)$ and $n \in[2,3)$. For $n \in(0,2)$, zero-contact-angle solutions are characterized by the so called entropy estimate (see $[11,15]$ in one space dimension, and [18, 16] for $N \leqslant 3$ )

$$
\begin{aligned}
\sup _{t \in(0, T)} \int_{\mathbb{R}^{N}} \zeta^{4} u(\cdot, t)^{\alpha+1} & +C^{-1} \int_{0}^{T} \int_{\mathbb{R}^{N}} \zeta^{4}\left|D^{2} u^{(\alpha+n+1) / 2}\right|^{2} \\
& \leqslant \int_{\mathbb{R}^{N}} \zeta^{4} u_{0}^{\alpha+1}+C \int_{0}^{T} \int_{\mathbb{R}^{N}} u^{\alpha+n+1}\left(|\nabla \zeta|^{4}+\zeta^{2}|\Delta \zeta|^{2}\right),
\end{aligned}
$$

which holds for all $\alpha \in(\max (0,1 / 2-n), 2-n)$ and all $\zeta \in C^{2}\left(\mathbb{R}^{N} ; \mathbb{R}_{0}^{+}\right)$. This estimate is also the starting point for results on waiting time. Indeed, if we take $\zeta \in \mathcal{F}\left[0, \theta, e_{N}\right]$ (which can easily be justified by an appropriate approximation argument) and choose

$$
w=u^{(\alpha+n+1) / 2}, \quad p=s=2, \quad q=\frac{2(\alpha+1)}{\alpha+n+1}, \quad k=l=2, \quad F\left(w_{0}\right)=w_{0}^{q},
$$

Theorem 2.1 applies with

$$
S(R)=\sup _{r \in(0, R)} r^{-4(\alpha+1) / n-N} \int_{\mathcal{C}(r)} u_{0}^{\alpha+1}
$$

Thus, part (1) recovers the critical growth exponent $\gamma^{*}=4 / n$ which has already been obtained in [19], and part (2) yields the lower bound

$$
T^{*} \geqslant C\left(\sup _{R>0} S(R)\right)^{-n /(\alpha+1)} .
$$

For initial data such that $u_{0}(x) \leqslant B|x|^{4 / n}$, Corollary 2.3 (with $\left.\mu=(\alpha+n+1) / 2\right)$ translates as

$$
T^{*} \geqslant C B^{-n} \text {. }
$$

In the parameter regime $n \in[2,3)$, entropies defined similarly to 3.5 are no longer globally decreasing. Therefore, we take advantage of the weighted energy estimate

$$
\sup _{t \in(0, T)} \int_{\mathbb{R}^{N}} \zeta^{6}|\nabla u(\cdot, t)|^{2}+C^{-1} \int_{0}^{T} \int_{\mathbb{R}^{N}} \zeta^{6}\left|\nabla u^{(n+2) / 6}\right|^{6} \leqslant \int_{\mathbb{R}^{N}} \zeta^{6}\left|\nabla u_{0}\right|^{2}+C \int_{0}^{T} \int_{[\zeta>0]} u^{n+2}
$$


for $\zeta \in \mathcal{F}\left[0, \theta, e_{N}\right]$, as derived in [27]. Together with a Hardy-type inequality valid on cones (see [28, Section 3]), we infer the inequality

$$
\sup _{t \in(0, T)} \int_{\mathbb{R}^{N}} \zeta^{4} u^{2}(\cdot, t)+C^{-1} \int_{0}^{T} \int_{\mathbb{R}^{N}} \zeta^{6}\left|\nabla u^{(n+2) / 6}\right|^{6} \leqslant \int_{\mathbb{R}^{N}} \zeta^{6}\left|\nabla u_{0}\right|^{2}+C \int_{0}^{T} \int_{[\zeta>0]} u^{n+2} .
$$

Note that the presence of a generic parameter $l$ in 2.6) is essential for Theorem 2.1 to apply in this case. Choosing

$$
w=u^{(n+2) / 6}, \quad p=s=6, \quad q=\frac{12}{n+2}, \quad k=1, \quad l=\frac{2}{3}, \quad F\left(w_{0}\right)=\left|\nabla w^{6 /(n+2)}\right|^{2},
$$

we obtain

$$
S(R)=\sup _{r \in(0, R)} r^{-2(4 / n-1)-N} \int_{\mathcal{C}(r)}\left|\nabla u_{0}\right|^{2} .
$$

Note that in this case the condition is stronger than in the previous one as it involves the gradient of initial data. Nevertheless, Theorem 2.1 still recovers the critical growth exponent $4 / n$ obtained already in [19, 28] and yields

$$
T^{*} \geqslant C\left(\sup _{R>0} S(R)\right)^{-n / 2}
$$

as the lower bound for the waiting time. For initial data satisfying the critical growth condition in the sense of

$$
\left|\nabla u_{0}(x)\right| \leqslant B|x|^{4 / n-1},
$$

inequality (3.8) translates again into the lower bound (3.6).

Theorem 2.1 also applies to doubly non-linear thin-film equations in one space dimension:

$$
u_{t}+\left(u^{n}\left|u_{x x x}\right|^{p-2} u_{x x x}\right)_{x}=0 .
$$

Existence and qualitative properties of zero-contact-angle solutions have been worked out in [2] for $p>2, n \in((p-1) / 2,2 p-1)$. In particular, the following counterpart of 3.7 can be found there:

$$
\sup _{t \in(0, T)} \int_{\mathbb{R}} \zeta^{3 p-2} u^{2}(\cdot, t)+C^{-1} \int_{0}^{T} \int_{\mathbb{R}} \zeta^{3 p}\left|\left(u^{(n+p) / p}\right)_{x x x}\right|^{p} \leqslant \int_{\mathbb{R}} \zeta^{3 p} u_{0 x}^{2}+C \int_{0}^{T} \int_{[\zeta>0]} u^{n+p}
$$

for all $\zeta \in \mathcal{F}\left[0, e_{N}, \theta\right]$. Applying Theorem 2.1 with

$$
w=u^{(n+p) / p}, \quad s=p, \quad q=\frac{2 p}{n+p}, \quad k=3, \quad l=\frac{3 p-2}{p},
$$

we get

$$
S(R)=\sup _{r \in(0, R)} r^{-2\left(\frac{3 p-2}{n+p-2}-1\right)-1} \int_{\mathcal{C}(r)} u_{0 x}^{2} .
$$

The critical growth exponent $(3 p-2) /(n+p-2)$ coincides with the one obtained in [2], and for initial data of the form

$$
\left|u_{0 x}(x)\right| \leqslant B|x|^{\frac{3 p-2}{n+p-2}-1}
$$

part (2) yields the lower bound

$$
T^{*} \geqslant C B^{-(n+p-2)}
$$




\section{Applications to degenerate parabolic systems}

This section is devoted to the analysis of waiting time phenomena for the following drift-diffusion model for semiconductors (see [30] and the references therein):

$$
\begin{cases}n_{t}=\operatorname{div}\left(\nabla n^{\alpha}-n \nabla V\right)-R(n, p) & \text { in } \Omega \times(0, T), \\ p_{t}=\operatorname{div}\left(\nabla p^{\alpha}+p \nabla V\right)-R(n, p) & \text { in } \Omega \times(0, T), \\ \Delta V=n-p-D(x) & \text { in } \Omega \times(0, T) .\end{cases}
$$

The unknowns $n$ and $p$ describe electron and hole density, respectively, $V$ is the electric potential and $R$ serves as a recombination term. We assume $\Omega \subset \mathbb{R}^{N}$ to be a bounded domain representing the semiconductor, the doping profile $D(\cdot)$ to be essentially bounded, and $\alpha>1$ (a typical example for $\alpha$ is $\alpha=5 / 3$ which corresponds to a high-injection regime, see [23] and the references therein). The problem is complemented by mixed Dirichlet / homogeneous Neumann boundary conditions and non-negative, bounded initial data:

$$
\begin{cases}n=n_{D}, \quad p=p_{D}, \quad V=V_{D} & \text { on }(\partial \Omega)_{D} \times(0, T), \\ \nabla n \cdot v=\nabla p \cdot v=\nabla V \cdot v=0 & \text { on }(\partial \Omega)_{N} \times(0, T), \\ n(x, 0)=n_{0}(x), \quad p(x, 0)=p_{0}(x) & \text { for } x \in \Omega .\end{cases}
$$

We assume the Dirichlet boundary data to be also bounded. For this system, subjected to mild assumptions on $R(\cdot, \cdot)$, Díaz, Galiano, and Jüngel established global existence of weak solutions in [22]. In addition, they proved the following result on the occurrence of a waiting time phenomenon.

LEMma (Díaz, Galiano, Jüngel) Assume that

$$
R(n, p) \geqslant b n^{\beta} \quad \text { for all } n, p \geqslant 0,
$$

with $b>0$ and $\beta \in(0,1)$ satisfying $\alpha+\beta<2$. Then the solution $(n, p, V)$ of (4.1) satisfies the following. Let $x_{0} \in \Omega, \varepsilon>0$ be sufficiently small and $0<\rho_{0}<\rho_{1}<\operatorname{dist}\left(x_{0}, \partial \Omega\right)$. If $n_{0} \equiv 0$ in $B\left(x_{0}, \rho_{0}\right)$ and if

$$
\sup _{\rho \in\left(\rho_{0}, \rho_{1}\right)}\left(\rho-\rho_{0}\right)^{-\gamma-N} \int_{B\left(x_{0}, r\right)} n_{0}^{\alpha+1} d x \leqslant \varepsilon, \quad \gamma=\frac{2(\alpha+1)}{\alpha-1},
$$

then there exists $T^{*}>0$ such that $n(\cdot, t)=0$ in $B\left(x_{0}, \rho_{0}\right)$ for all $0<t<T^{*}$.

In what follows, we will show that it is also possible to derive a lower bound on the waiting time. As a by-product, our result shows that

- the condition $\alpha+\beta<2$ may be relaxed to $\beta<1$, removing the constraints on $\alpha(\alpha<2-\beta<2)$ and $\beta(\beta<2-\alpha)$;

- it is not necessary to impose any smallness condition on $\varepsilon$,

- the occurrence of a waiting time phenomenon may be established at each point of $\partial B\left(x_{0}, \rho_{0}\right)$ rather than only globally on $B\left(x_{0}, \rho_{0}\right)$.

It turns out that local results on finite speed of propagation only depend on global quantities like $\|n\|_{\infty},\|p-n-D\|_{\infty}$, and the distance from the boundary of the initial support. This fact allows us to apply Theorem 2.1 to the more general situation of mixed Dirichlet/homogeneous Neumann boundary conditions. Our result holds for connected components $A$ of $\operatorname{supp}\left(n_{0}\right)$ which are compact subsets of $\Omega$, and reads as follows. 
Proposition 4.1 Assume (4.3) holds for some $\beta \in(0,1)$. Let $(n, p, V)$ be a solution of (4.1)(4.2) as obtained in [22], and let $A$ be a connected component of $\operatorname{supp}\left(n_{0}\right)$ such that

$$
\begin{aligned}
& A \subset B\left(x_{0}, R_{0}\right) \subset B\left(x_{0}, R_{0}+5 d\right) \subset \Omega, \\
& A=\operatorname{supp}\left(n_{0}\right) \cap B\left(x_{0}, R_{0}+5 d\right)
\end{aligned}
$$

for some $x_{0} \subset \mathbb{R}^{N}, R_{0}>0$ and $d>0$. Let

$$
\begin{aligned}
T_{0} & =\sup \left\{t \in(0, T): t\|n-p-D\|_{L^{\infty}(\Omega \times(0, T))} \leqslant 1 / 4\right\}, \\
\tilde{\alpha} & =\min \{\alpha, 2-\beta\}>1 .
\end{aligned}
$$

Then:

(a) $T_{1}=\sup \left\{t \leqslant T_{0}: n(\cdot, t) \equiv 0\right.$ in $\left.B\left(x_{0}, R_{0}+3 d\right) \backslash B\left(x_{0}, R_{0}+d\right)\right\}>0$;

(b) if furthermore $A$ has the "exterior cone property" at $z_{0} \in \partial A$, i.e. $A \cap \operatorname{supp}\left(\zeta\left[z_{0}, \theta, a\right]\right)=\emptyset$ for some $\theta \in(0, \pi / 2), a \in S^{n-1}$, and if for some $\delta \in(1,3]$ we have

$$
\lim _{r \downarrow 0} r^{-\gamma-N} \int_{\mathcal{C}\left[z_{0}+r a, \theta, a\right] \cap A} n_{0}^{\delta}<\infty, \quad \gamma=\frac{2 \delta+N(\alpha-\tilde{\alpha})}{\tilde{\alpha}-1},
$$

then $n$ has positive waiting time at $z_{0}$;

(c) if furthermore

$$
S=\sup _{r>0} r^{-\gamma-N} \int_{\mathcal{C}\left[z_{0}+r a, \theta, a\right] \cap A} n_{0}^{\delta}<\infty,
$$

then the waiting time $T^{*}$ is estimated from below as

$$
T^{*} \geqslant \min \left\{T_{1},\left(C_{1} S\right)^{-\frac{2(\tilde{\alpha}-1)}{2 \delta+N(\alpha-\tilde{\alpha})}}\right\}
$$

where $C_{1}$ depends on $\theta, N, \alpha, \beta, b, \delta,\|n\|_{L^{\infty}(\Omega \times(0, T))},\|n-p-D\|_{L^{\infty}(\Omega \times(0, T))}$, and $d$.

Here, the range $\delta \in(1,3]$ has been chosen such that, under the assumption $\alpha+\beta<2$ of the Díaz-Galiano-Jüngel Lemma, $\delta=\alpha+1$ is always admissible in (4.8). In that case the two critical exponents $\gamma$ in (4.4), resp. (4.8), coincide-whereas the results do not, since (4.8) is a local condition at $z_{0}$.

Proof. For notational convenience, we introduce the sets

$$
B(R)=B\left(x_{0}, R\right) .
$$

Let us start by taking a generic cut-off $\zeta$ compactly supported in $B\left(R_{0}+4 d\right)$. A few integrations by parts show that for $\delta>1$,

$$
\begin{aligned}
\int_{\Omega} \zeta^{2} n^{\delta}(\cdot, T)= & \int_{\Omega} \zeta^{2} n_{0}^{\delta}-\alpha \delta \int_{0}^{T} \int_{\Omega} n^{\alpha+\delta-2} \nabla\left(\zeta^{2}\right) \nabla n \\
& +\int_{0}^{T} \int_{\Omega} n^{\delta} \nabla\left(\zeta^{2}\right) \nabla V-(\delta-1) \int_{0}^{T} \int_{\Omega} \zeta^{2} n^{\delta} \Delta V \\
& -\alpha \delta(\delta-1) \int_{0}^{T} \int_{\Omega} \zeta^{2} n^{\alpha+\delta-3}|\nabla n|^{2}-\delta \int_{0}^{T} \int_{\Omega} \zeta^{2} n^{\delta-1} R(n, p)
\end{aligned}
$$


In view of $4.5 p, \operatorname{dist}\left(B\left(R_{0}+4 d\right), \partial \Omega\right) \geqslant d$. Hence, by standard results of elliptic regularity theory we infer the existence of a constant $C$, depending on $\|n-p-D\|_{L^{\infty}\left(\Omega \times\left(0, T_{0}\right)\right)}$ and $d$, such that

$$
\|\nabla V\|_{L^{\infty}\left(B\left(R_{0}+4 d\right) \times\left(0, T_{0}\right)\right)} \leqslant C .
$$

Combining (4.3) and 4.9 with standard absorption methods entails, for $T \leqslant T_{0}$,

$$
\begin{aligned}
\sup _{t \in(0, T)} \int_{\Omega} \zeta^{2} n^{\delta}(\cdot, t)+ & C^{-1} \int_{0}^{T} \int_{\Omega} \zeta^{2} n^{\alpha+\delta-3}|\nabla n|^{2}+C^{-1} \int_{0}^{T} \int_{\Omega} \zeta^{2} n^{\delta+\beta-1} \\
\leqslant & \int_{\Omega} \zeta^{2} n_{0}^{\delta}+(\delta-1) T_{0}\|n-p-D\|_{L^{\infty}\left(\Omega \times\left(0, T_{0}\right)\right)} \sup _{t \in(0, T)} \int_{\Omega} \zeta^{2} n^{\delta}(\cdot, t) \\
& +C \int_{0}^{T} \int_{\Omega}|\nabla \zeta|^{2}\left(n^{\alpha+\delta-1}+n^{\delta+1-\beta}\right) .
\end{aligned}
$$

Using the definition (4.6) of $T_{0}$ and $\delta \in(1,3]$, we thus obtain in particular

$$
\begin{aligned}
\sup _{t \in(0, T)} \int_{\Omega} \zeta^{2} n^{\delta}(\cdot, t)+C^{-1} \int_{0}^{T} \int_{\Omega} \zeta^{2} n^{\alpha+\delta-3}|\nabla n|^{2} & \\
& \leqslant 2 \int_{\Omega} \zeta^{2} n_{0}^{\delta}+C \int_{0}^{T} \int_{\Omega}|\nabla \zeta|^{2}\left(n^{\alpha+\delta-1}+n^{\delta+1-\beta}\right) .
\end{aligned}
$$

We may further simplify the right-hand side provided we also allow $C$ to depend on $\|n\|_{L^{\infty}\left(\Omega \times\left(0, T_{0}\right)\right)}$. Then, with $\tilde{\alpha}$ as in 4.7 , we conclude that

$$
\sup _{t \in(0, T)} \int_{\Omega} \zeta^{2} n^{\delta}(\cdot, t)+C^{-1} \int_{0}^{T} \int_{\Omega} \zeta^{2} n^{\alpha+\delta-3}|\nabla n|^{2} \leqslant \int_{\Omega} \zeta^{2} n_{0}^{\delta}+C \int_{0}^{T} \int_{\Omega}|\nabla \zeta|^{2} n^{\tilde{\alpha}+\delta-1}
$$

As is well known (see e.g. [16], [21]), using arguments similar to those of Theorem 1-with test functions $\zeta_{r}$ supported on nested balls rather than on nested cones-4.10 yields the following local result on finite speed of propagation:

Lemma 4.2 Assume that $n_{0} \equiv 0$ on $B\left(x_{1}, r_{1}\right) \subset B\left(R_{0}+4 d\right)$. Then there exists a continuous, decreasing function $r(t)$, independent of $x_{1}$, such that $r(0)=r_{1}$ and

$$
n(\cdot, t) \equiv 0 \quad \text { on } B\left(x_{1}, r(t)\right) .
$$

Combining Lemma 4.2 with 4.5 shows that there is a positive time $T_{1}$ such that

$$
n(\cdot, t) \equiv 0 \quad \text { in } B\left(R_{0}+3 d\right) \backslash B\left(R_{0}+d\right) \quad \text { for all } t \in\left(0, T_{1}\right) .
$$

Indeed, it is sufficient to cover $B\left(R_{0}+4 d\right) \backslash B\left(R_{0}\right)$ by balls of radius $2 d$ centered on $\partial B\left(R_{0}+2 d\right)$ and to apply the lemma above. As long as $t<T_{1}$, we may thus extend $\left.n(\cdot, t)\right|_{B\left(R_{0}+3 d\right)}$ by zero to the whole of $\mathbb{R}^{N}$ for each $t \in\left(0, T_{1}\right)$. If we introduce, for $t<T_{1}$,

$$
w(x, t)= \begin{cases}n^{(\alpha+\delta-1) / 2}(x, t), & x \in B\left(R_{0}+3 d\right), \\ 0, & \text { elsewhere }\end{cases}
$$


(4.10) may be reformulated as

$$
\sup _{t \in(0, T)} \int_{\mathbb{R}^{N}} \zeta^{2} w^{q}(\cdot, t)+C^{-1} \int_{0}^{T} \int_{\mathbb{R}^{N}} \zeta^{2}|\nabla w|^{2} \leqslant \int_{\mathbb{R}^{N}} \zeta^{2} w_{0}^{q} d x+C \int_{0}^{T} \int_{[\zeta>0]}|\nabla \zeta|^{2} w^{s}
$$

for all $\zeta$ supported in $B\left(R_{0}+3 d\right)$, with

$$
q=\frac{2 \delta}{\alpha+\delta-1}, \quad s=\frac{2(\tilde{\alpha}+\delta-1)}{\alpha+\delta-1} .
$$

But in view of 4.11 , $w(\cdot, t) \equiv 0$ on $\mathbb{R}^{N} \backslash B\left(R_{0}+d\right)$ for all $0<t<T_{1}$. Hence the restriction $\operatorname{supp}(\zeta) \subset B\left(R_{0}+3 d\right)$ is not necessary any longer and $(4.12)$ holds for test functions in $\mathcal{F}\left[z_{0}, \theta, a\right]$. If we observe that $|\nabla \zeta| \leqslant C(\theta)$ and that $q<s$ (since $\tilde{\alpha}>1$ ), an application of Theorem 2.1 (with $p=2, k=l=1$ ) now completes the proof of Proposition 4.1 Note that the presence of a generic $s$ in 2.6 is essential for Theorem 2.1 to apply to this case.

\section{Acknowledgements}

L.G. acknowledges the kind hospitality of the Institut für Angewandte Mathematik in Bonn. This work was supported by E.C. through the RTN-Programme "Fronts-Singularities" (HPRN-CT2002-00274), by DFG through SFB 611 "Singuläre Phänomene und Skalierung in mathematischen Modellen" at the University of Bonn, and by G.N.A.M.P.A through the project "Interfacce e frontiere libere in problemi parabolici degeneri di ordine superiore". Finally, we would like to thank Herbert Koch for pointing out to us the impact of parabolic Harnack inequalities on quantitative waiting time estimates for the porous-medium equation.

\section{REFERENCES}

1. Alikakos, N. D. On the pointwise behavior of solutions of the porous medium equation as $t$ approaches zero or infinity. Nonlinear Anal. 9 (1985), 1095-1113. Zbl 0589.35064 MR 0806912

2. Ansini, L., \& Giacomelli, L. Doubly nonlinear thin-film equations in one space dimension. Arch. Rat. Mech. Anal. 173 (2004), 89-131. Zbl 1064.76012 MR 2073506

3. Antontsev, S. N. On the localization of solutions of nonlinear degenerate elliptic and parabolic equations. Soviet Math. Dokl. 24 (1981), 420-424. Zbl 0496.35051

4. Antontsev, S. N., \& DíAZ, J. I. On space or time localization of solutions of nonlinear elliptic and parabolic equations via energy methods. Recent Advances in Nonlinear Elliptic and Parabolic Problems (Nancy, 1988), Pitman Res. Notes Math. Ser. 208, Longman Sci. Tech., Harlow (1989), 3-14. Zbl 0696.35090 MR 1034991

5. Antontsev, S. N., DíAz, J. I., \& Shmarev, S. I. Energy Methods for Free Boundary Problems. Applications to Nonlinear PDEs and Fluid Mechanics. Birkhäuser, Boston (2002). Zbl 0988.35002 MR 1858749

6. Antontsev, S. N., Díaz, J. I., \& Shmarev, S. I. The support shrinking in solutions of parabolic equations with non-homogeneous absorption terms. Elliptic and Parabolic Problems (Pont-à-Mousson, 1994), Pitman Res. Notes Math. Ser. 325, Longman Sci. Tech., Harlow (1995), 24-39. Zbl 0828.35065 MR 1416571

7. Aronson, D. G., \& CAfFarelli, L. A. The initial trace of a solution of the porous medium equation. Trans. Amer. Math. Soc. 280 (1983), 351-366. Zbl 0556.76084 MR 0712265 
8. Aronson, D. G., Caffarelli, L. A., \& Kamin, S. How an initially stationary interface begins to move in the porous medium flow. SIAM J. Math. Anal. 14 (1983), 639-658. Zbl 0542.76119 MR 0704481

9. Andreucci, D., \& Tedeev, A. F. Finite speed of propagation for the thin-film equation and other higher-order parabolic equations with general nonlinearity. Interfaces Free Bound. 3 (2001), 233-264. Zbl 1002.35059 MR 1843587

10. Bénilan, P., Crandall, M. G., \& Pierre, M. Solutions of the porous medium equation in $\mathbb{R}^{N}$ under optimal conditions on initial values. Indiana Univ. Math. J. 33 (1984), 51-87. Zbl 0552.35045 MR 0726106

11. Beretta, E., Bertsch, M., \& Dal Passo, R. Nonnegative solutions of a fourth order nonlinear degenerate parabolic equation. Arch. Rat. Mech. Anal. 129 (1995), 175-200. Zbl 0827.35065 MR 1328475

12. BERNIS, F. Existence results for "doubly" nonlinear higher order parabolic equations on unbounded domains. Math. Ann. 279 (1988), 373-394. Zbl 0609.35048 MR 0922422

13. BERnis, F. Qualitative properties for some nonlinear higher order degenerate parabolic equations. Houston J. Math. 14 (1988), 319-352. Zbl 0682.35009 MR 0985928

14. Bernis, F. Finite speed of propagation for thin viscous flows when $2 \leqslant n<3$. C. R. Acad. Sci. Paris Sér. I Math. 322 (1996), 1169-1174. Zbl 0853.76018 MR 1396660

15. Bertozzi, A. L., \& Pugh, M. The lubrication approximation for thin viscous films: regularity and long time behavior of weak solutions. Comm. Pure Appl. Math. 49 (1996), 85-123. Zbl 0863.76017 MR 1371925

16. Bertsch, M., Dal Passo, R., Garcke, H., \& GrüN, G. The thin viscous flow equation in higher space dimensions. Adv. Differential Equations 3 (1998), 417-440. Zbl 0954.35035 MR 1751951

17. Chipot, M., \& Sideris, T. An upper bound for the waiting time for nonlinear degenerate parabolic equations. Trans. Amer. Math. Soc. 288 (1985), 423-427. Zbl 0535.35049 MR 0773069

18. Dal PAsso, R., Garcke, H., \& GRÜN, G. On a fourth order degenerate parabolic equation: global entropy estimates and qualitative behavior of solutions. SIAM J. Math. Anal. 29 (1998), 321-342. Zbl 0929.35061 MR 1616558

19. Dal Passo, R., Giacomelli, L., \& GRÜN, G. A waiting time phenomenon for thin film equations. Ann. Scuola Norm. Sup. Pisa 30 (2001), 437-463. Zbl 1024.35051 MR 1895718

20. Dal Passo, R., Giacomelli, L., \& Grün, G. Waiting time phenomena for degenerate parabolic equations-a unifying approach. Geometric Analysis and Nonlinear Partial Differential Equations, S. Hildebrandt and H. Karcher (eds.), Springer (2003), 637-648. Zbl 1028.35078 MR 2008360

21. Dal Passo, R., Giacomelli, L., \& Shishkov, A. The thin film equation with nonlinear diffusion. Comm. Partial Differential Equations 26 (2001), 1509-1557. Zbl 1001.35070 MR 1865938

22. DíAZ, J. I., GAliAnO, G., \& JÜNGEL, A. On a quasilinear degenerate system arising in semiconductor theory. Part I: Existence and uniqueness of solutions. Nonlinear Anal. Real World Appl. 2 (2001), 305-336. Zbl 0994.35072 MR 1835610

23. DÍAZ, J. I., GAliAnO, G., \& JÜNGEL, A. On a quasilinear degenerate system arising in semiconductor theory. Part II: Localization of vacuum solutions. Nonlinear Anal. 36 (1999), 569-594. Zbl 0924.35066 MR 1673262

24. Gagliardo, E. Ulteriori proprietà di alcune classi di funzioni in più variabili. Ricerche Mat. 8 (1959), 24-51. Zbl 0199.44701 MR 0109295

25. Giacomelli, L., \& Shishrov, A. E. Propagation of support in one-dimensional convected thin-film flow. Indiana Univ. Math. J. 54 (2005), 1181-1215. Zbl pre02228533 MR 2164423

26. GRÜN, G. Droplet spreading under weak slippage: a basic result on finite speed of propagation. SIAM J. Math. Anal. 34 (2003), 992-1006. Zbl 1035.35049 MR 1969611 
27. GrüN, G. Droplet spreading under weak slippage: existence for the Cauchy problem. Comm. Partial Differential Equations 29 (2004), 1697-1744. Zbl pre02171338 MR 2105985

28. GRÜN, G. Droplet spreading under weak slippage: the waiting time phenomenon. Ann. Inst. H. Poincaré Anal. Non Linéaire 21 (2004), 255-269. Zbl 1062.35012 MR 2047357

29. GRÜN, G. On free boundary problems arising in thin film flow. Habilitation thesis, Univ. Bonn (2001).

30. JÜNGEL, A. Asymptotic analysis of a semiconductor model based on Fermi-Dirac statistics. Math. Methods Appl. Sci. 19 (1996), 401-424. Zbl 0860.35008 MR 1376513

31. Knerr, B. F. The porous medium equation in one dimension. Trans. Amer. Math. Soc. 234 (1977), 381-415. Zbl 0365.35030 MR 0492856

32. Nirenberg, L. On elliptic partial differential equations. Ann. Scuola Norm. Sup. Pisa 13 (1959), 115162. Zbl 0088.07601 MR 0109940

33. Nirenberg, L. An extended interpolation inequality. Ann. Scuola Norm. Sup. Pisa 20 (1966), 733-737. Zbl 0163.29905 MR 0208360

34. SAPronov, D. A., \& ShishKov, A. E. Propagation of perturbations in quasilinear multidimensional parabolic equations with convective term. Ukrainian Math. J. 53 (2001), 1134-1155. Zbl 0989.35067 MR 2031292

35. Shishkov, A. E. On estimates of the speed of propagation of perturbations in quasilinear divergent degenerate parabolic equations of higher order. Ukrainian Math. J. 44 (1992), 1335-1340. Zbl 0799.35104 MR 1201147

36. Shishkov, A. E., \& Shchelkov, A. G. Dynamics of the support of energy solutions of mixed problems for quasi-linear parabolic equations of arbitrary order. Izv. Math. 62 (1998), 601-626. Zbl 0918.35075 MR 1642176

37. Stampacchia, G. Équations elliptiques du second ordre à coefficients discontinus. Les Presses de l‘Univ. de Montréal, Montréal (1966). Zbl 0151.15501 MR 0251373 